\title{
3D Numerical study on the effect of shaft eccentricity on the tribological performance of lubricated sliding contact
}

\author{
Mohammad Tauviqirrahman", Bayu Kurniawan, Jamari \\ Laboratory for Engineering Design and Tribology, Mechanical Engineering Department, Engineering \\ Faculty, Diponegoro University, Indonesia
}

\begin{abstract}
Recently, a growing interest is given to the wall slip and the artificial texturing for improving the performance of lubricated sliding contact. The use of wall slip, artificial texturing, and the combination of slip and texturing can be the effective approach to enhance the performance of the bearing. The present study examines the effect of shaft eccentricity ratio on the hydrodynamic journal bearing performance. 3D numerical modelling based on modified Reynolds equation is used to analyse the effect of texturing and the wall slip on the characteristics of a hydrodynamically lubricated sliding contact. The analysis results point out that with respect to the load support and the power loss of the bearing, the use of wall slip on smooth surface is the most excellent configuration compared to other patterns (i.e. slip-texturing, pure texturing and conventional patterns). It is also confirmed that the wedge effect due to the shaft eccentricity has a significant role in altering the lubricant behaviour. Thus, a particular care must be taken in choosing the pattern of lubricated sliding contact as well as the shaft eccentricity.
\end{abstract}

\section{Introduction}

As a mechanical element, journal bearing is designed to support shaft or journal which rotates freely in a supporting metals or shell. In order to avoid direct contact between the surfaces, the insertion of a lubricant into the region around the interacting devices is carried out, so wear can be reduced. Due to the divergent-convergent gap the pressure gradients that exist in the clearance between the shaft and the housing produce the load support. Recently, along with the development of advanced technology and the measurement, the artificial texturing concept as well as the wall slip concept in various configurations is introduced and explored by numerous researchers in the world focusing on how to improve the hydrodynamic lubrication performance.

The superiority of artificially textured surface over the smooth surface in terms of load support and friction force has been examined by several researchers. Cupillard et al. [1] confirmed that textured journal bearing is superior to smooth journal bearing with thinner

\footnotetext{
*Corresponding author : mohammad.tauviqirrahman@ft.undip.ac.id
} 
lubricant with respect to the friction force. A comparative study between textured surface and smooth surface of non-recessed hybrid journal bearing configurations was also conducted by Khatri and Sharma [2]. They found that the textured journal bearing provides an improved stability parameter than that of smooth journal bearing. With respect to the texture location, based on the computational fluid dynamic (CFD), Liang et al. [3] investigated the effect of partial texture location and texture depth on load support, friction coefficient and circumferential flow of journal bearing. They revealed that the texture located at leading edge of the contact could enhance the bearing performance, and the effect of shallow dimples is superior to the deep ones. Regarding with the effect of texture shape, Sinanoğlu et al. [4] studied the effect of trapezoidal texture on the performance of journal bearing. They concluded that journal with trapezoidal texture pattern can support more loads than the sawtooth texture.

Recently, in addition to artificial texturing, the wall slip is also considered as a method to improve the lubrication performance. Numerically, $\mathrm{Wu}$ [5] proposed the potential of wall slip application to maximize the load support. Wang et al. [6] conducted the optimization of the wall slip region of the combined surface sleeve bearing. Later, the idea of combining the artificial texturing with wall slip has also attracted many researchers. Tauviqirrahman et al. [7] explored the characteristics of the textured/wall slip surface with respect to the load support. It is found that in comparison with a solely textured surface, the load support of the combined textured/wall slip pattern can be increased by around $300 \%$. Later, Aurelian et al. [8] revealed that a particular care should be taken in choosing the texture/slip pattern because an inappropriate choice can lead to a significant decrease in the performance.

In the present work, in order to extend the insight of previously published findings, the artificial texturing and wall slip pattern are considered as a base of design of the journal bearing. Several surface modes of the lubricated sliding contact containing different patterns of the artificial texturing and wall slip (i.e. slip, textured, and textured-wall slip conditions) are investigated. The main aim of the present study is to investigate the effect of shaft eccentricity (i.e. the wedge effect) on the tribological performance for different surface configurations. Here, modified Reynolds equation is developed considering the wall slip surface and solved by means of finite volume method.

\section{Analysis}

As is known in classical literature, the derivation of traditional Reynolds theory is based on assumption of no-slip between the lubricant and the contacting surfaces. The lubrication model presented here is based on the fact that slip in the lubricant will exist in the interface. Schematic of lubricated sliding contacts with artificial texturing and slip/no slip condition is shown in Figure 1.

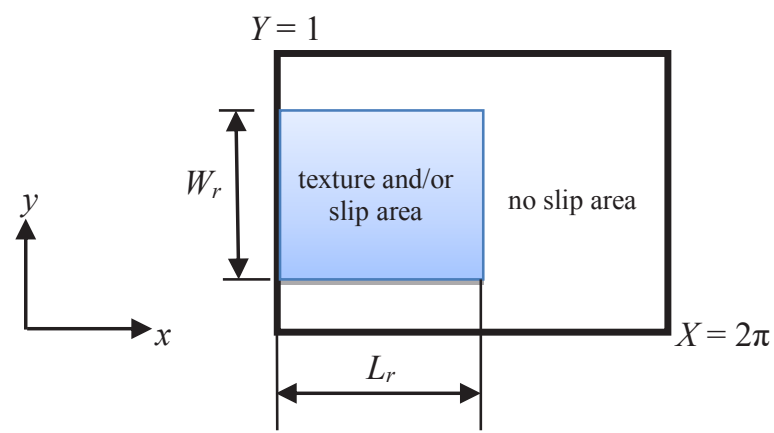

Fig. 1. Schematic representation of lubricated sliding contact with partial texturing and/or wall slip (Note: $W_{r}=0.7, L_{r}=170^{\circ}$ ) 
In the present work, the artificial texturing zone as well as the wall slip is introduced at the leading edge of the contact. It is defined by $W_{r} \times L_{r}$ zone where $W_{r}$ is the width of the modified zone and $L_{r}$ is the length of modified zone. The numerical analysis is carried out by analysing the behaviour of fluid induced by several patterns studied here. In the developed model, the slip has been treated to allow in certain areas where the shear stress exceeds a critical value. When both criteria are met, the resulting slip velocity is proportional to the difference between the shear stress and the critical value, with constant factors referred as $\alpha$. Such a lubrication system can be described with solving the modified Reynolds equation as follows [7]:

$$
\begin{array}{r}
\frac{\partial}{\partial x}\left(h^{3} \frac{\partial p}{\partial x}\left(1+\frac{3 \alpha \eta}{h+\alpha \eta}\right)\right)+\frac{\partial}{\partial y}\left(h^{3} \frac{\partial p}{\partial y}\left(1+\frac{3 \alpha \eta}{h+\alpha \eta}\right)\right) \\
=\frac{\partial}{\partial x}\left(-6 \eta \frac{\alpha \tau_{c}}{h+\alpha \eta} h^{2}+6 u_{w}\left(1+\frac{\alpha \eta}{h+\alpha \eta}\right)\right)
\end{array}
$$

The physical meanings of the symbols in Equation (1) are as follows: $h$ the lubrication film thickness (gap) at location, $p$ the lubrication film pressure, $u_{w}$ the sliding velocity, $\eta$ the lubricant viscosity, $\alpha$ the slip coefficient and $\tau_{c}$ the critical shear stress of the interface. It can be seen in that if the slip length $\alpha$ and $\tau_{c}$ are set to zero (no-slip condition), Equation (1) reduces to the classical Reynolds equation.

In the present work, the finite volume method combined with tridiagonal matrix algorithm (TDMA) and alternating direction implicit (ADI) method is used to address the nonlinear governing Reynolds equation for lubricated sliding contact based on the critical shear stress model. The Reynolds boundary condition is used to modelling the cavitation. In order to obtain the highest positive effect of the wall slip, the critical shears stress $\tau_{c}$ is set to zero $[9,10]$.

The primary parameters of the lubricated sliding contact are given as follows: journal rotation is $5100 \mathrm{rpm}$, total length of bearing is $535.6 \times 10^{-3} \mathrm{~m}$, journal radius is $209.7 \times 10^{-3} \mathrm{~m}$, bearing radius is $210.25 \times 10^{-3} \mathrm{~m}$, clearance is $0.55 \times 10^{-3} \mathrm{~m}$, dynamic viscosity is 0.02785 Pa.s and it is assumed that fluid density is $855.6 \mathrm{~kg} / \mathrm{m}^{3}$. In this study, for modeling the optimal slip characteristic, the dimensionless slip length $A$ of 10 is considered.

\section{Results and Discussion}

For analysis, 3D lubrication model is developed. In this way, the lubricant behaviour in the $y$-direction is taken into account to get more realistic result based on the work of [9]. In the present study, the rectangular shape is chosen as a layout for texturing as well as the wall slip. The load support and the power loss are of main particular interest as an indicator of the lubrication performance.

Figure 2 shows the effect of shaft eccentricity ratio on the power loss varying different surface characteristics. Specific features can be identified based on Figure 2. Firstly, for the case of no-slip situation, introducing the artificial texturing on lubricated sliding contact has advantage with respect to the power loss. However, when the eccentricity ratio is increased, the power loss increases significantly and the performances of bearing with texturing is worse than the smooth conventional bearing. It can be seen that for the case of eccentricity ratio $\varepsilon$ which is larger than 0.7 , the power loss increases significantly with the increase of the eccentricity ratio. Secondly, for the case of slip condition, the lubricated contact with or 
without texture produces lower power loss compared to the contact with no-slip condition either with texture or without texture. Figure 2 also shows that the best pattern in producing the lowest power loss in lubricated sliding contact is smooth slip surface.

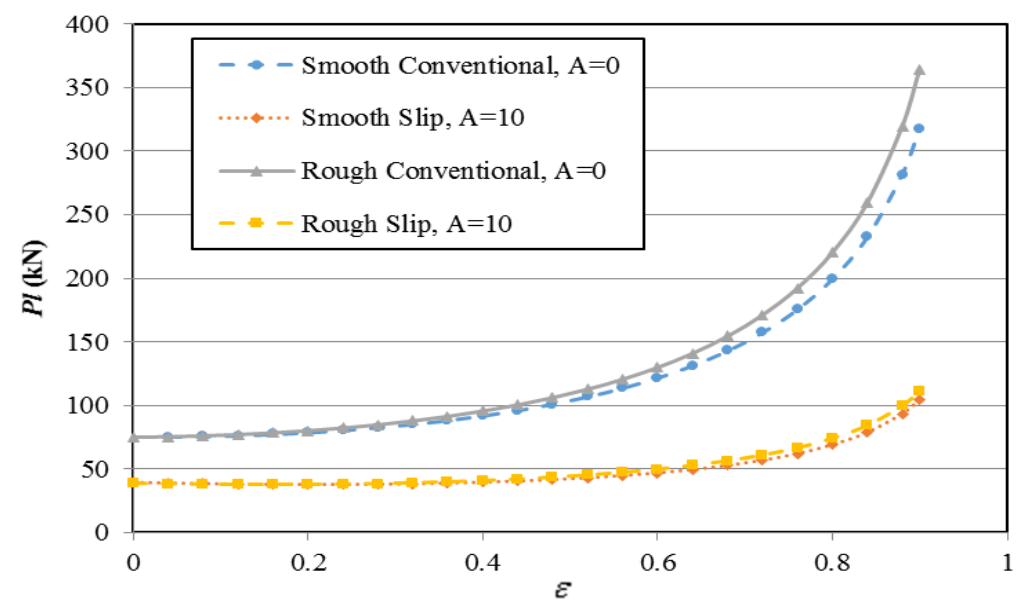

Fig. 2. Effect of the shaft eccentricity ratio $\varepsilon$ on the power loss $P l$

Figure 3 reflects the effect of eccentricity ratio on the load support for different surface configurations. It can be found that the eccentricity ratio has a significant influence on the load support for all configurations. Increasing eccentricity ratio improves the load support significantly especially for high eccentricity ratio ( $\varepsilon>0.8$ in this case). It can also be observed that introducing the modification of contacting surface either with artificial texturing or the wall slip leads to better performance compared to smooth surface. It is obvious in Figure 3 that for $\varepsilon<0.5$ the rough slip pattern (which refers to the combined texturing and wall slip) generates higher load support than others. However, for higher eccentricity ratio, the smooth slip is the best pattern in generating the high support.

Figure 3 also shows that the introduction of wall slip as well as the artificial texturing in journal bearing will be useless if high eccentricity ratio is used. It means that for high eccentricity ratio $(\varepsilon>0.8$ in this case), the predicted load support of smooth traditional surface is higher than that of other surfaces. This finding is in a good agreement with the work of [8] for case of slider bearing. It seems that the wedge ratio has more dominant role in altering the lubricant characteristics compared to the texturing and/or slip technique. In detail, the limit of effect of eccentricity ratio on the load support $W$ for several patterns considered here is shown in Table 1. Based on this table, it is recommended that for the case of high eccentricity ratio, the smooth surface (without slip) is preferable for real application. 


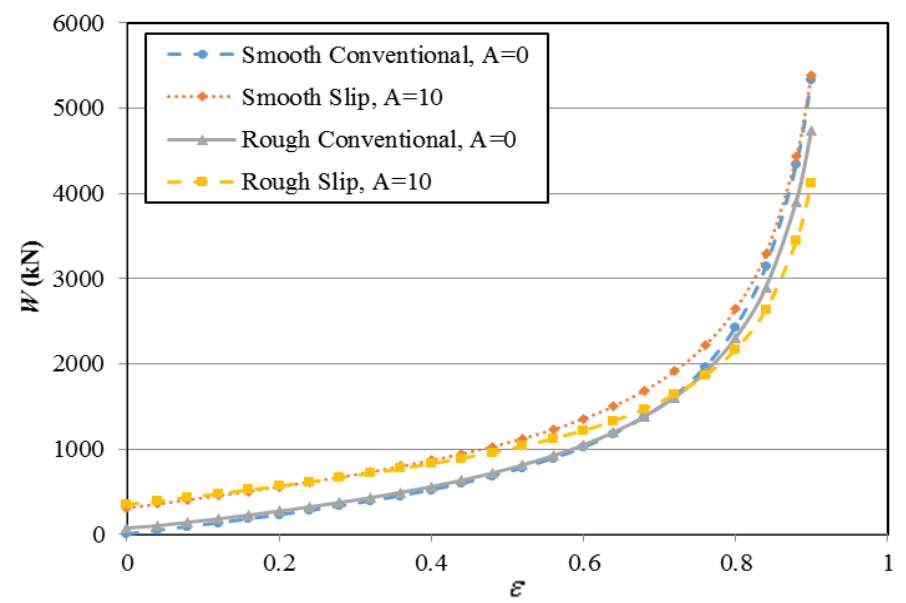

Fig. 3. Effect of the shaft eccentricity ratio $\varepsilon$ on the load support $W$

Table 1. Limit of eccentricity ratio

\begin{tabular}{|c|c|}
\hline Model & $\varepsilon_{\text {lim }}$ \\
\hline Smooth Slip & 0.90 \\
\hline Rough Conventional & 0.72 \\
\hline Rough Slip & 0.72 \\
\hline
\end{tabular}

Note: $\varepsilon_{\lim }$ is the eccentricity ratio of the modified journal bearing which generates the same load support with that of traditional journal bearing

\section{Conclusion}

In the present work, the lubricant behaviour of journal bearing with several configurations (i.e. slip-texturing, pure texturing and conventional patterns) was explored. The effect of the eccentricity ratio on the load support and the power loss of journal bearing is of particular interest. The following conclusions summarize the results of the present study:

1. The eccentricity ratio has a significant effect in altering the lubricant characteristic for all patterns studied here.

2. Introduction of wall slip as well as artificial texturing on the journal bearing is quite effective in improving the lubrication performance especially for case of low eccentricity ratio.

3. The superiority of smooth slip surface is highlighted in terms of the power loss and load support compared to textured surfaces for all range of eccentricity ratios.

\section{References}

1. S. Cupillard, S. Glavatskih, M.J. Cervantes, Proc. Inst. Mech. Eng. Part J: J. Eng. Tribol. 222, 96 (2008).

2. C.B. Khatri, S.C Sharma, Tribol. Int. 95, 221 (2016). 
3. X. Liang, Z. Liu, H. Wang, X. Zhou, X. Zhou, Ind. Lubr. Tribol. 68, 106 (2016).

4. C. Sinanoğlu, F. Nair, M.B. Karamiş, J. Mater. Process. Technol. 168, 344 (2005).

5. C. Wu, Ind. Lubr. Tribol. 60, 293 (2008).

6. L.L. Wang, C.H. Lu, M. Wang, W.X Fu, Tribol. Int. 47, 100 (2012).

7. M. Tauviqirrahman, Muchammad, Jamari, D.J. Schipper, Tribol. Trans. 57, 134 (2014).

8. F. Aurelian, M. Patrick, H. Mohamed, Tribol. Int. 44, 868 (2011).

9. M. Tauviqirrahman, R. Ismail, Jamari, D.J. Schipper, Comput. Fluids 79, 27 (2013).

10.F. Aurelian, M. Patrick, H. Mohamed, Tribol. Int. 44, 868 (2011). 